Die Zukunft der Nachhaltigkeitsberichterstattung I/IV \section{Von der Kür zur Pflicht}

\author{
Nachhaltigkeitsberichterstattung wird mal als Bedingung \\ effektiven Nachhaltigkeitsmanagements gesehen, mal als \\ PR-Masche. In einer vierteiligen Serie beleuchtet \\ ÖkologischesWirtschaften den Status quo sowie mögliche \\ Zukunftsszenarien. In Teil 1 fordert die Autorin verpflichtende \\ Nachhaltigkeitsberichterstattung nach internationalen \\ Standards. Von Susanne Bergius
}

$S^{t}$ ellen Sie sich vor, Sie hielten einen Bericht von 30 Seiten in den Händen mit allen entscheidenden Informationen zu einem Unternehmen: zur Geschäftsentwicklung sowie zu Risiken, Chancen und Leistungen hinsichtlich Umwelt, Sozialem und Unternehmensführung (internationales Kürzel: ESG). Kein Ballast mehr durch dicke Finanzberichte und $\mathrm{zu}$ detaillierte Informationen.

Qualität statt Quantität - Verfechter einer integrierten Berichterstattung wollen die Firmenberichterstattung revolutionieren hin zu einem Werkzeug, das bessere Entscheidungen bewirken kann. Doch wer entscheidet, was wichtig ist? In Geschäftsberichten fehlen oft wesentliche ESG-Informationen, weil das Management sie nicht als solche erkennt. Ein Mechanismus muss sicherstellen, dass Firmen finanzielle und ESG-Aspekte verbinden.

\section{Reicht „Integrated Reporting"?}

Nein, denn es bedient Kapitalmarktakteure, die mit Nachhaltigkeit Wirtschaftskrisen besser meistern wollen. Selbst die meisten Research-Akteure zu Nachhaltigkeit nutzen ökonomisch orientierte Konzepte. Mitarbeiter(innen), Kund(innen)en und die Gesellschaft können ihre berechtigten ökosozialen Ansprüche folglich nicht so gut durchsetzen.

Um diese Gruppen zu informieren, bleibt eine breitere Nachhaltigkeitsberichterstattung unerlässlich. Zudem ent- faltet sie ihre größte Wirkung nicht bei der externen Kommunikation, sondern intern: Sie sensibilisiert Mitarbeiter(innen), bewegt Abteilungen zu kooperieren, legt Schwachstellen und Potenziale offen und ist oft der Anstoß für eine Nachhaltigkeitsstrategie.

Was braucht eine zukunftsfähige Nachhaltigkeitsberichterstattung? Soll sie ernst genommen werden, müssen sich Unternehmen an international anerkannten Standards orientieren, die Vergleiche mit Wettbewerbern ermöglichen. Eine gute Basis bieten die Kriterien der Global Reporting Initiative, dem einzigen durch einen Multistakeholder-Dialog erarbeiteten und international anerkannten Standard.

Unternehmen sollten darstellen, wie ihre Nachhaltigkeitsstrategien auf das Kerngeschäft ausgerichtet sind, welche anspruchsvollen Ziele und Zeitvorgaben bestehen, ob ein wirksames Nachhaltigkeitsmanagement mit klaren Verantwortlichkeiten existiert und welche ökosozialen Entscheidungskriterien für Investitionen etabliert sind. Umwelt- und Sozialleistungen sind transparent darzustellen, mit mehrjährigen Zeitreihen, möglichst extern geprüft und standardisiert. Zahlen allein reichen aber nicht: Überdies sind die Zusammenhänge zwischen ökonomisch-finanziellen und ökologisch-sozialen Leistungen klarzumachen. Glaubwürdig ist, wer Ziele und Erreichtes abgleicht und kritisch reflektiert. Den meisten Firmen fehlt dazu der Mut.

\section{Führt Freiwilligkeit zum Ziel?}

Offensichtlich nicht, denn erst 1.000 der 42.000 Konzerne in der Europäischen Union (EU) betreiben „Corporate Social Responsibility“ (CSR) strategisch, nur 2.500 berichten überhaupt. Darum plant die EU-Kommission eine verbindliche Berichterstattung für Konzerne. Dann müssen sich auch die mit CSR befassen, die ihre Verantwortung bisher ignorieren. Protest kommt nur aus Deutschland: Mehrere Bundesministerien und die Wirtschaftsverbände BDI, BDA und DIHK blocken ab.

Warum aber erachten viele die Pflicht zur Finanzberichterstattung als selbstverständlich, nicht aber eine Regel, nach der Unternehmen offenlegen müssen, ob und wie sie negative Auswirkungen ihres Kerngeschäfts auf Umwelt und Gesellschaft mindern?

\section{Fazit}

Ob man eine ESG-Berichtspflicht mag oder nicht, sie wird kommen - und sei es über den Druck von Investoreninitiativen auf Unternehmen, Börsen und Politik. So haben im Mai Großanleger und globale Verbände, hinter denen 30 Billionen Dollar Vermögen stehen, vor dem Rio+ 20-Erdgipfel von den Regierungen der Welt ESG-Berichtspflichten gefordert. Solche bestehen bereits in Dänemark, Frankreich, Südafrika und Brasilien. Diese Länder wollen sich weltweit dafür einsetzen, dass Nachhaltigkeitsberichterstattung zum Standard wird.

Es hat hundert Jahre gebraucht, bis sich die Finanzberichterstattung etablierte - ein vergleichbares Reporting $\mathrm{zu}$ Nachhaltigkeit wird sich viel schneller durchsetzen.

\section{AUTORIN + KONTAKT}

Susanne Bergius ist unabhängige Journalistin und Moderatorin für Nachhaltiges Wirtschaften und Investieren. Sie schreibt für Tages- und Fachmedien und ist seit 2008 zuständig für das „Handelsblatt Business Briefing Nachhaltige Investments“.

Susanne Bergius, Blumenthalstr. 21, 12103 Berlin, Tel.: +49 3093953309

E-Mail: susanne.bergius@gmx.com Internet: www.handelsblatt.com/nachhaltigkeit 
Copyright (C) 2012, IÖW und oekom Verlag. Die Nutzung des Artikels ist Abonnenten von Ökologisches Wirtschaften vorbehalten. Nachdruck und Vervielfältigung des Artikels einschließlich Speicherung und Nutzung auf optischen und elektronischen Datenträgern nur mit Zustimmung der Redaktion von Ökologisches Wirtschaften (http://www.oekologischeswirtschaften.de). 